\title{
SECAGEM EM CAMADA DELGADA DA FIBRA RESIDUAL DO MARACUJÁ
}

\author{
ROSINELSON DA SILVA PENA* \\ NILSON BELO MENDONÇA**
}

\begin{abstract}
Estudou-se a secagem em camada delgada do resíduo da industrialização do suco de maracujá (Passiflora edulis), em diferentes condições de temperatura $\left(50 \mathrm{a} 70^{\circ} \mathrm{C}\right)$ e velocidade do ar de secagem $(2,0$ a $3,0 \mathrm{~m} / \mathrm{s})$. Foram obtidas isotermas de adsorção e dessorção de umidade a 25 e $40^{\circ} \mathrm{C}$, visando avaliar o comportamento higroscópico do produto. Avaliou-se a aplicabilidade das equações de Kuhn, Handerson, Oswin, Halsey, Smith, GAB e BET na predição dos dados de sorção. A adsorção indicou que o produto estará microbiologicamente estável $\left(a_{w}<0,6\right)$ quando apresentar umidade inferior a $18 \mathrm{~g} / 100 \mathrm{~g}$ (b.s). A monocamada indicou que o resíduo não deve ser seco a umidades inferiores a 7,4 $\mathrm{g} \mathrm{H}_{2} \mathrm{O} / 100 \mathrm{~g}$ (b.s). para evitar desperdício de energia. Os calores de dessorção indicaram que a maior parte da água adsorvida no produto está combinada aos solutos. As equações de Kuhn, Oswin, Halsey, GAB e BET podem ser utilizadas na predição das isotermas de sorção de umidade do produto.
\end{abstract}

PALAVRAS-CHAVE: Passiflora edulis; MARACUJÁ - RESÍDUO; SECAGEM EM CAMADA DELGADA; ISOTERMAS.

* Professor, Doutor em Engenharia Química, Faculdade de Engenharia de Alimentos, Instituto de Tecnologia, Universidade Federal do Pará (UFPA) Belém, PA, Brasil (e-mail: rspena@ufpa.br).

** Engenheiro Químico pela UFPA, Belém, PA, Brasil (e-mail: nilsoneq@yahoo.com.br). 


\section{INTRODUÇÃO}

O Brasil se destaca no mercado internacional pela produção de maracujá devido a atributos como extensão de área, clima privilegiado, relevo pouco acidentado e inúmeros outros fatores favoráveis à cultura (OLIVEIRA et al., 2002).

Os maiores produtores mundiais de maracujá localizam-se na América do sul, sendo o Brasil, a Colômbia, o Peru e o Equador os maiores exportadores, tendo como principal produto o suco concentrado (SABBAG et al., 2002). De acordo com o último censo, o estado do Pará é o quarto maior produtor nacional, sendo superado apenas pelos estados da Bahia, do Ceará e de Sergipe (IBGE, 2006).

Entre as indústrias alimentícias instaladas no estado do Pará merece destaque a indústria de beneficiamento de frutas que gera elevado volume de rejeito industrial (AYROSA et al., 2007). Constituído de cascas, sementes e bagaços, esses rejeitos, por apresentarem elevado teor de açúcares, estão muito susceptíveis ao desencadeamento de processos fermentativos, exalando mau cheiro nos lugares de descarga e servindo de foco para a presença de animais (SOUZA e SANDI, 2001). Praticamente todo o resíduo gerado é descartado, sendo apenas uma parte doada para pequenos criadores de gado, nas proximidades das indústrias (SILVA, 2002).

Um dos principais alvos da indústria de alimentos é encontrar formas de aproveitamento dos resíduos gerados, que possam ser revertidos em benefícios financeiros a fim de minimizar ou até evitar impactos ambientais (RUGGIERO, 1996). Um dos processos alternativos que pode se utilizado com tal finalidade é a secagem (OKADA, 1987; AKPINAR, 2006).

A secagem (operação unitária) proporciona a remoção da água de um sólido na forma de vapor para a fase gasosa insaturada. Ocorre por mecanismo de vaporização térmica em temperatura inferior à de ebulição da água (FERRUA e BARCELOS, 2003). Na secagem de alimentos, a água (umidade) é removida empregando-se o ar como fase gasosa insaturada (GEANKOPLIS, 1983; McCABE et al., 1993).

O rejeito gerado na produção do suco de maracujá apresenta características próprias, podendose destacar a presença de macronutrientes (como açúcares, proteínas e fibras), além de micronutrientes. Essas características permitem o desenvolvimento de aplicabilidade industrial alimentar para o resíduo gerado, incluindo a composição de produtos matinais e barras; o enriquecimento de produtos alimentícios, principalmente quanto ao teor de fibras como ração animal, adubo ou matéria-prima para a extração de pectina, que se apresenta em quantidade considerável, principalmente no mesocarpo do maracujá (BUCKERIDGE e TINÉ, 2001).

Estudou-se a secagem em leito fixo (camada delgada) visando o aproveitamento do resíduo da industrialização do suco de maracujá, produzido por indústria instalada no estado do Pará, bem como o comportamento higroscópico do produto obtido para estabelecer suas condições de secagem e armazenamento.

\section{MATERIAL E MÉTODOS}

\subsection{MATÉRIA-PRIMA}

Utilizou-se como matéria-prima o segundo resíduo (fibra residual) do beneficiamento do suco de maracujá amarelo (Passiflora edulis f. flavicarpa), fornecido pela indústria NOVA AMAFRUTAS, localizada no município de Benevides (PA). O resíduo foi adquirido congelado e mantido a $-18^{\circ} \mathrm{C}$ até sua utilização.

\subsection{CARACTERIZAÇÃO DO RESÍDUO}

Caracterizou-se o resíduo físico-quimicamente pelas determinações de: umidade em estufa a $105^{\circ} \mathrm{C}$; proteínas totais pela determinação do nitrogênio total, utilizando-se o fator de conversão de 6,25; resíduo mineral fixo (cinzas) por calcinação a $550^{\circ} \mathrm{C}$; gordura (extrato etéreo) por extração com éter de petróleo e fibra alimentar total pelo método enzimático (AOAC, 1997). 


\subsection{SECAGEM DO RESÍDUO}

\subsubsection{Obtenção dos dados de secagem}

O resíduo congelado foi submetido ao descongelamento em ambiente com temperatura média de $5^{\circ} \mathrm{C}$ (refrigerador) e prensado manualmente para eliminar parte da fração líquida. Testes preliminares indicaram que a utilização do resíduo prensado proporcionou produto menos pastoso após secagem, além de favorecer a formação e manutenção do leito no secador. Após a prensagem, o resíduo foi homogeneizado para ser submetido aos ensaios de secagem em secador de leito fixo (Figura 1), com variação de temperatura na câmara de $\pm 1^{\circ} \mathrm{C}$.

\section{FIGURA 1 - ESQUEMA DO SECADOR DE LEITO FIXO UTILIZADO}

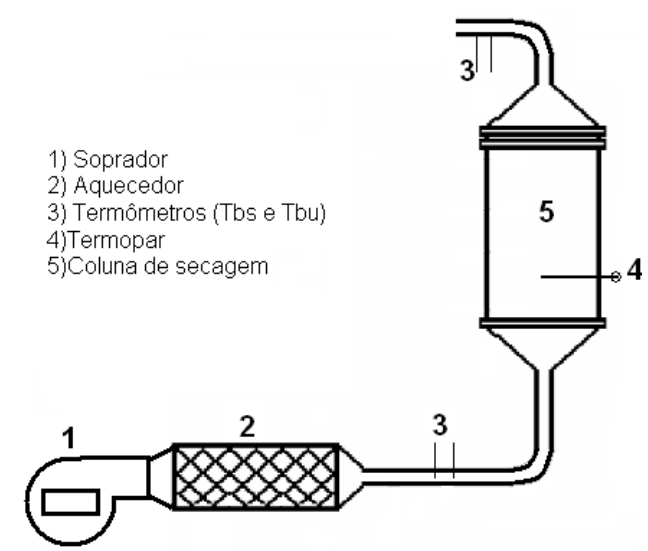

Os ensaios de secagem foram realizados nas temperaturas $(\mathrm{T})$ de 50,60 e $70^{\circ} \mathrm{C}$, com velocidade do ar de secagem $\left(V_{a r}\right)$ de 2,0, 2,5 e 3,0 m/s. Utilizou-se planejamento experimental do tipo quadrático com ponto central, tendo como variáveis de entrada $T$ e $V_{\mathrm{ar}}$ e de resposta a umidade no equilíbrio $\left(X_{e}\right)$, o tempo total de secagem ( $\left.t_{\text {total }}\right)$ e a taxa de secagem no período constante $\left(W_{c}\right)$. Pesou-se aproximadamente $100 \mathrm{~g}$ do resíduo em bandeja perfurada, com área de contato de $378,0 \mathrm{~cm}^{2}$. O material foi espalhado homogeneamente sobre a superfície da bandeja para formar camada delgada.

A pesagem das amostras durante a secagem foi feita a cada 2 minutos nos 10 primeiros minutos; a cada 5 min até 30 minutos; a cada 10 min até 60 minutos e a cada 20 minutos a partir desse tempo. Não havendo mais variação na massa da amostra (peso constante), parte da mesma foi retirada para determinação do peso seco em balança de infravermelho. A aplicabilidade dessa metodologia foi validada comparando-se os resultados obtidos com os da secagem em estufa a $105^{\circ} \mathrm{C}$. A metodologia e o tratamento de dados foram baseados em WAUGHON e PENA (2008).

\subsubsection{Estudo da cinética de secagem}

Para avaliar a cinética de secagem foram construídas curvas de taxa de secagem (MARTINAZZO et al., 2007). A área utilizada para calcular a taxa de secagem foi o tamanho da bandeja do secador $\left(378,0 \mathrm{~cm}^{2}\right)$, sendo construídas curvas de taxa de secagem em função da umidade e do tempo, utilizando-se a Equação 1. Os valores de $\mathrm{d} \mathrm{X}_{\mathrm{bs}} / \mathrm{dt}$ foram obtidos a partir das derivadas das equações da reta (Equação 2) e de Midilli (MADAMBA, DRISCOLL e BUCKLE, 1996), substituindo-se a umidade reduzida (MR) pela umidade em base seca $\left(X_{b s}\right)$ (Equação 3). As equações foram ajustadas aos dados 
experimentais de secagem ( $X_{b s}$ versus $\left.t\right)$ de acordo com o comportamento da relação, linear ou não, com o auxílio do aplicativo STATISTICA ... (2000).

$$
\begin{gathered}
W_{T}=\frac{m_{s}}{A} \cdot\left[-\frac{d X_{b s}}{d t}\right] \\
X_{b s}=a+b \cdot t \\
X_{b s}=b^{\prime} \cdot t+c \cdot e^{-k \cdot t^{n}}
\end{gathered}
$$

Em que:

$\mathrm{X}_{\mathrm{bs}}=$ umidade (g/100 g b.s); $\mathrm{t}=$ tempo $(\mathrm{min}) ; \mathrm{W}_{\mathrm{T}}=$ taxa de secagem $\left(\mathrm{g} / \mathrm{cm}^{2} . \mathrm{h}\right) ; \mathrm{m}_{\mathrm{s}}=$ peso seco; $A$ = área da superfície exposta a secagem; $a, b, b$, c, k e $n=$ constantes.

\subsection{AVALIAÇÃO HIGROSCÓPICADO PRODUTO}

\subsubsection{Obtenção dos dados de sorção}

Na obtenção dos dados de adsorção, amostras do produto $(1 \pm 0,0010 \mathrm{~g})$ foram pesadas em recipientes do aparelho medidor de atividade de água e submetidas à secagem complementar em dessecador com sílica gel sob vácuo e a $25^{\circ} \mathrm{C}$, durante 24 horas. Em seguida, foram colocadas em dessecador contendo água na base e o conjunto levado para ambiente na temperatura de trabalho.

Para a obtenção dos dados de dessorção, as mesmas amostras foram mantidas no dessecador contendo água na base na temperatura de trabalho por 24 horas para que ocorresse a umidificação das mesmas. Em seguida, foram transferidas para dessecador com sílica gel e o conjunto acondicionado em ambiente na temperatura de trabalho. Nos dois processos, as amostras foram retiradas em duplicata em tempos crescentes (equilíbrio dinâmico) para determinação da umidade por diferença de massa e da atividade de água em higrômetro AQUAlab 3TE da Decagon.

Os dados de adsorção e dessorção foram obtidos a 25 e $40^{\circ} \mathrm{C}$, utilizando-se estufa para DBO com variação de temperatura de $\pm 1^{\circ} \mathrm{C}$. Essa metodologia foi testada por ASSUNÇÃO e PENA (2007) e SILVA, SILVA e PENA (2008), sendo utilizada pelo grupo de alimentos da Universidade Federal do Pará com excelente reprodutibilidade. Durante todos os ensaios de sorção, as amostras foram submetidas à inspeção visual para acompanhamento de alterações perceptíveis, como perda de fluidez, escurecimento e crescimento de fungo.

\subsubsection{Determinação da monocamada}

A umidade de monocamada $\left(m_{0}\right)$ foi determinada pela equação de BET linearizada (Equação 4) (BRUNAUER, EMMET e TELLER 1938):

$$
\frac{a_{w}}{\left(1-a_{w}\right) \cdot m}=\frac{1}{m_{o} \cdot c}+\frac{(c-1)}{m_{o} \cdot c} \cdot a_{w}
$$

Em que:

$\mathrm{m}=$ umidade,

$\mathrm{a}_{\mathrm{w}}=$ atividade de água; $\mathrm{m}_{\mathrm{o}}=$ umidade de monocamada; e $\mathrm{C}=$ constante relacionada com o calor de adsorção. 


\subsubsection{Cálculo do calor de dessorção}

O calor isostérico integral de dessorção $\left(Q_{\mathrm{st}}\right)$ foi determinado pela Equação 5. A entalpia de vaporização da água $\left(\Delta \mathrm{H}_{\mathrm{v}}\right)$ foi obtida a partir de tabelas de pressão de vapor e o calor isostérico líquido de dessorção $\left(\mathrm{q}_{\mathrm{s}}\right)$ pela Equação 6 , forma integrada da equação de Clausius-Clayperon (ASSUNÇÃO e PENA, 2007; SILVA, SILVA e PENA, 2008):

$$
\begin{gathered}
\mathrm{Q}_{\mathrm{st}}=\mathrm{q}_{\mathrm{st}}+\Delta \mathrm{H}_{\mathrm{v}} \\
\ln \left(\frac{\mathrm{a}_{\mathrm{W}_{1}}}{\mathrm{a}_{\mathrm{W}_{2}}}\right)=\frac{-\mathrm{q}_{\mathrm{st}}}{\mathrm{R}} \cdot\left(\frac{1}{\mathrm{~T}_{1}}-\frac{1}{\mathrm{~T}_{2}}\right)
\end{gathered}
$$

\subsubsection{Predição das isotermas de sorção}

Foram testados sete modelos matemáticos, conforme descrito na Tabela 1.

\section{TABELA 1 - MODELOS UTILIZADOS NA PREDIÇÃO DAS ISOTERMAS DE

\begin{tabular}{|c|c|c|}
\hline Equação & Modelos & Referência \\
\hline Kuhn $^{\mathrm{a}}$ & $\mathrm{m}=-\frac{\mathrm{a}}{\ln \mathrm{a}_{\mathrm{w}}}+\mathrm{b}$ & CHIRIFE e IGLESIAS (1978) \\
\hline Handerson $^{a}$ & $m=\left[\frac{-\ln \left(1-a_{w}\right)}{a}\right]^{\frac{1}{b}}$ & CHIRIFE e IGLESIAS (1978) \\
\hline Oswin ${ }^{a}$ & $m=a\left[\frac{a_{w}}{1-a_{w}}\right]$ & CHIRIFE e IGLESIAS (1978) \\
\hline Halsey $^{a}$ & $\mathrm{~m}=\left[\frac{-\mathrm{a}}{\ln \mathrm{a}_{\mathrm{w}}}\right]^{\overline{\mathrm{b}}}$ & CHIRIFE e IGLESIAS (1978) \\
\hline Smith $^{\mathrm{a}}$ & $m=a-b \cdot \ln \left(1-a_{w}\right)$ & CHIRIFE e IGLESIAS (1978) \\
\hline $\mathrm{GAB}^{\mathrm{b}}$ & $m=\frac{m_{0} \cdot c \cdot k \cdot a_{w}}{\left[\left(1-k \cdot a_{w}\right) \cdot\left(1+(c-1) \cdot k \cdot a_{w}\right)\right]}$ & MAROULIS et al. (1988) \\
\hline $\mathrm{BET}^{\mathrm{c}}$ & $m=\frac{m_{0} \cdot c \cdot a_{w}}{1-a_{w}} \cdot\left(\frac{1-(n+1) \cdot a_{w}^{n}+n \cdot a_{w}^{n+1}}{1-(1-c) \cdot a_{w}-c \cdot a_{w}^{n+1}}\right)$ & PARK e NOGUEIRA (1992) \\
\hline
\end{tabular} SORÇÃO DO PRODUTO}

$\mathrm{m}=$ umidade; $\mathrm{a}_{\mathrm{w}}=$ atividade de água; $\mathrm{m}_{\mathrm{o}}=$ umidade monocamada; $\mathrm{a}, \mathrm{b}, \mathrm{c}, \mathrm{k}, \mathrm{n}=$ constantes.

Realizou-se a análise de regressão com auxílio do aplicativo STATISTICA ... (2000), utilizando a metodologia de estimativa de Levenberg-Marquardt e critério de convergência de $10^{-6}$. Os parâmetros utilizados para avaliar os ajustes foram o coeficiente de determinação $\left(r^{2}\right)$ e o desvio médio relativo $(P)$ (Equação 7). Valores de P menores que 10\%, considerados por PENG et al. (2007) como indicadores de bons ajustes para isotermas de sorção para propósitos práticos, foram tomados como referência para avaliar os ajustes. 


$$
P=\frac{100}{n} \sum_{i=1}^{n} \frac{\left|m_{\text {exp }}-m_{\text {pre }}\right|}{m_{\text {exp }}}
$$

Em que:

$\mathrm{m}_{\mathrm{exp}}$ e $\mathrm{m}_{\text {pre }}=$ umidades experimental e predita, respectivamente, e $\mathrm{n}=$ número de observações.

\section{RESULTADOS E DISCUSSÕES}

\subsection{CARACTERIZAÇÃO DO PRODUTO}

O resíduo, após secagem, apresentou como composição média: 10\% de umidade, 10,9\% de proteínas, 3,9\% de lipídios, 2,0\% de cinzas, 56,5\% de fibra alimentar total e teor de sólidos solúveis de $16,7 \%$. Destaca-se o teor excepcional de fibra alimentar que permite classificar o produto como rico em fibra, de acordo com a legislação brasileira (BRASIL, 1998), que exige no mínimo $6 \mathrm{~g}$ de fibras/ $100 \mathrm{~g}$ (para alimentos sólidos) para tal classificação.

\subsection{SECAGEM DO RESÍDUO}

\subsubsection{Curvas de secagem}

Curvas de secagem, obtidas a partir dos dados experimentais, são apresentadas nas Figuras 2 a 4 . Observou-se para todas as condições de secagem estudadas que a umidade final do produto foi superior a $10 \mathrm{~g} / 100 \mathrm{~g}$ b.s. Esse comportamento pode ser justificado pelo alto teor de sólidos solúveis (açúcares) do resíduo, os quais apresentam elevada afinidade por moléculas de água em função do processo de solvatação.

\section{FIGURA 2 - CURVAS DE SECAGEM DO RESÍDUO NA VELOCIDADE DO AR DE SECAGEM $2,0 \mathrm{~m} / \mathrm{s}$}

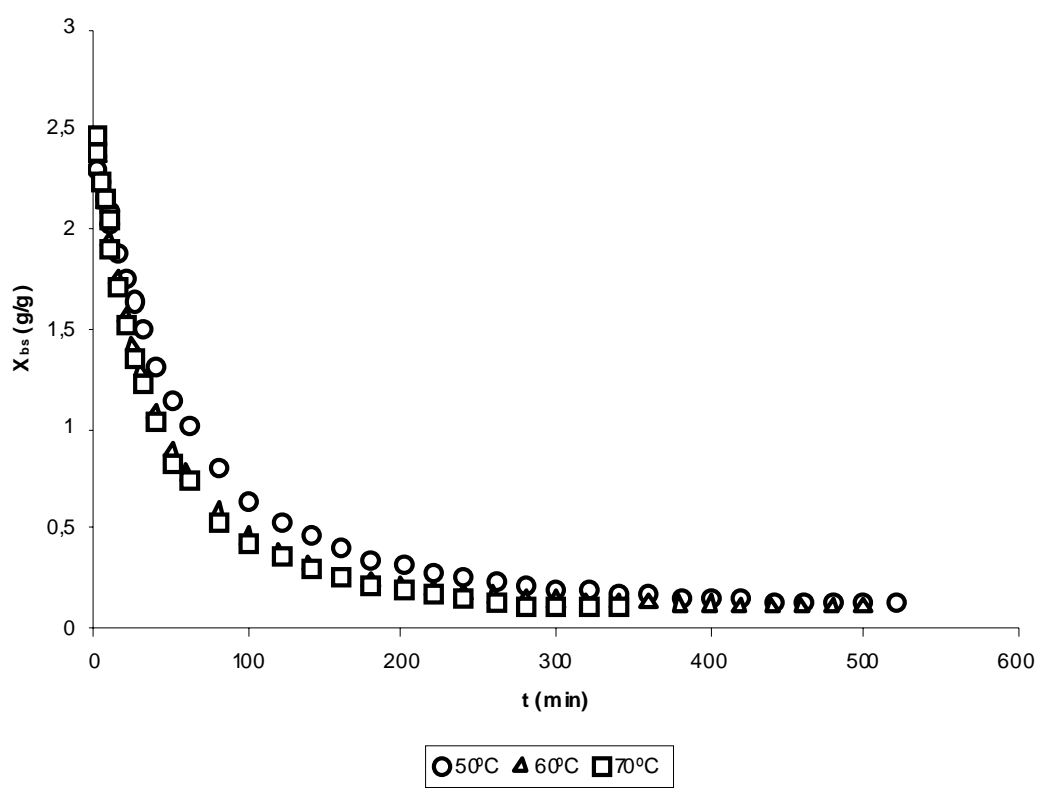




\section{FIGURA 3 - CURVAS DE SECAGEM DO RESÍDUO NA VELOCIDADE DO AR \\ DE SECAGEM $3,0 \mathrm{~m} / \mathrm{s}$}

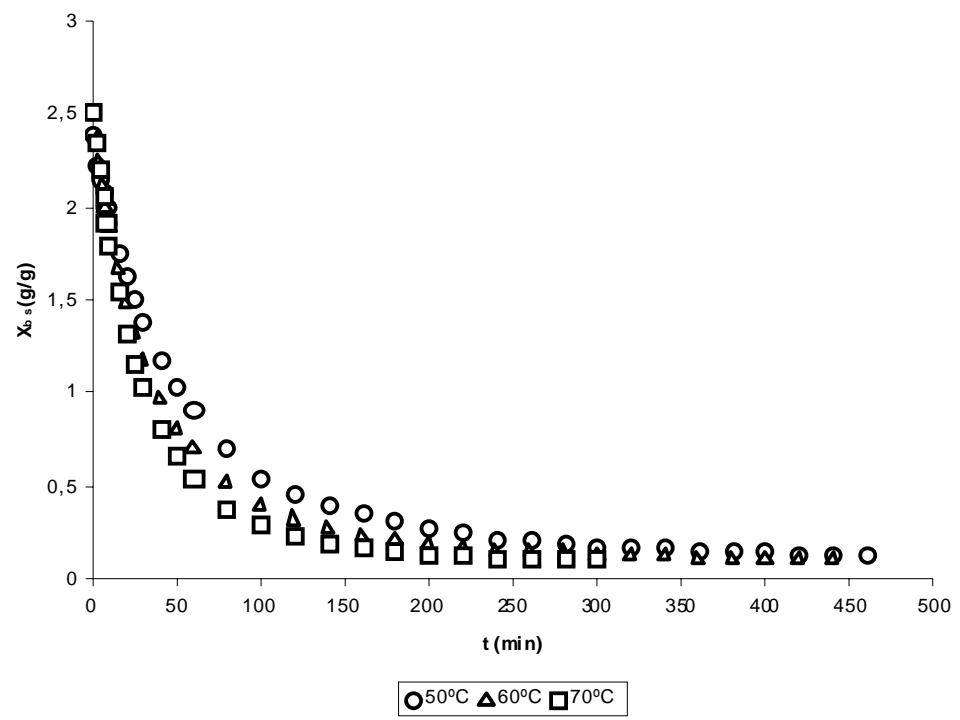

FIGURA 4 - CURVAS DE SECAGEM DO RESÍDUO NA TEMPERATURA DE 60 C

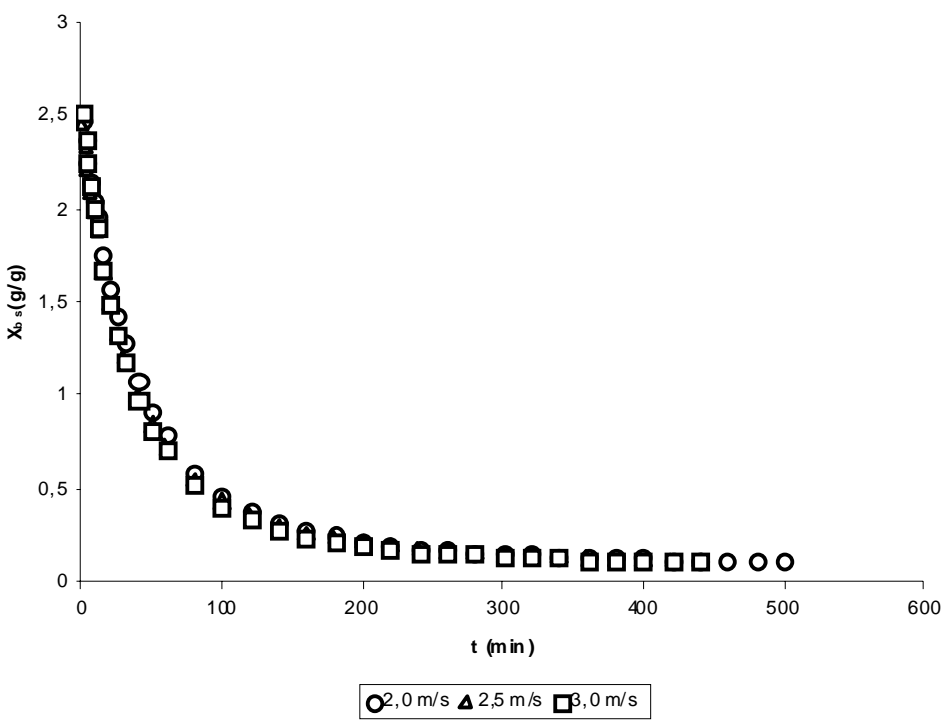

\subsubsection{Cinética de secagem}

Curvas de taxas de secagem $\left(\mathrm{W}_{\mathrm{T}}\right)$ para o produto, em função do tempo (t) e da umidade $\left(\mathrm{X}_{\mathrm{bs}}\right)$, são apresentadas nas Figuras 5 e 6 . Em todas as condições empregadas, observou-se curto período de secagem à velocidade constante, o qual se reduziu com o aumento da temperatura. Esse comportamento confirma que apenas pequena fração da água de constituição do resíduo se encontra na forma livre e que a maior fração interage com os solutos (sólidos solúveis).

\subsubsection{Avaliação estatística da secagem}

A análise de variância (ANOVA) aplicada aos dados de secagem indicou que: (1) a temperatura $(T)$ exerceu influência significativa linear $(p<0,001)$ e quadrática $(p<0,01)$ sobre a umidade no equilíbrio (Figura 7); (2) T exerceu influência linear ( $p<0,001)$ e quadrática $(p<0,01)$ e a velocidade do ar de 
secagem $\left(V_{a r}\right)$ também linear $(p<0,01)$ e quadrática $(p<0,05)$ sobre o tempo de secagem (Figura 8$)$; e (3) T exerceu influência linear $(p<0,001)$ e quadrática $(p<0,05)$ e $V_{a r}$ linear $(p<0,01)$ sobre $a$ taxa de secagem a velocidade constante (Figura 9 ). Com base nos efeitos das variáveis independentes sobre as variáveis dependentes, sugere-se, como boa condição de secagem para o resíduo em camada delgada, a temperatura de $65^{\circ} \mathrm{C}$ e velocidade do ar de secagem de $2,5 \mathrm{~m} / \mathrm{s}$. Nessas condições, o produto atingirá umidade de $11 \mathrm{~g} / 100 \mathrm{~g}$ b.s. em aproximadamente 6 horas de secagem.

\section{FIGURA 5 - CINÉTICA DE SECAGEM DO RESÍDUO NA VELOCIDADE DO AR DE SECAGEM $2,5 \mathrm{~m} / \mathrm{s}$}

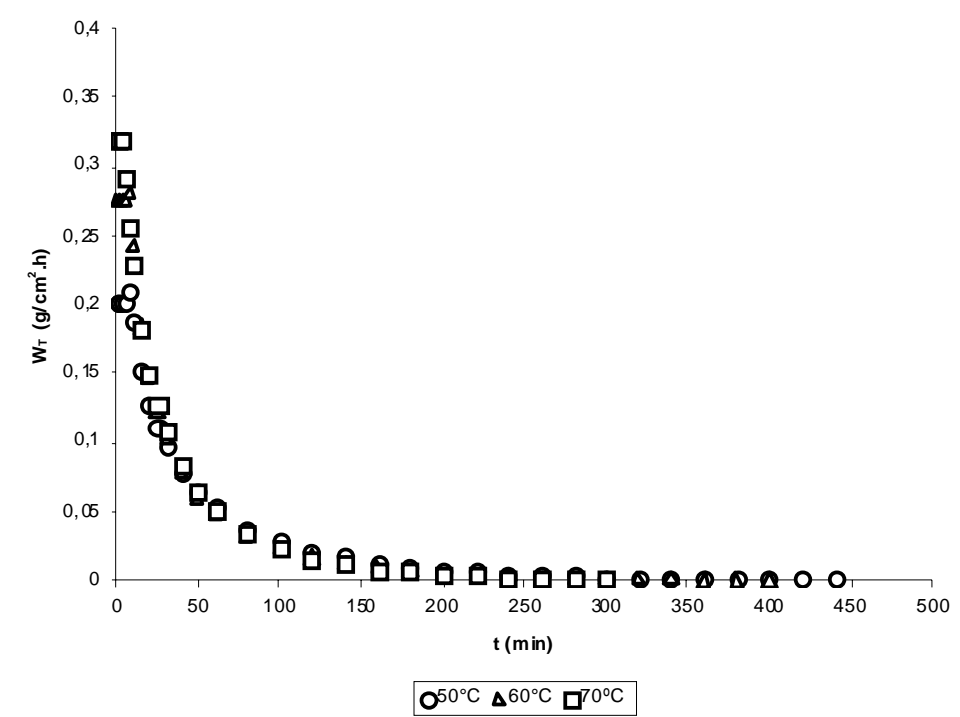

FIGURA 6 - CINÉTICA DE SECAGEM DO RESÍDUO NA VELOCIDADE DO AR DE SECAGEM $3,0 \mathrm{~m} / \mathrm{s}$

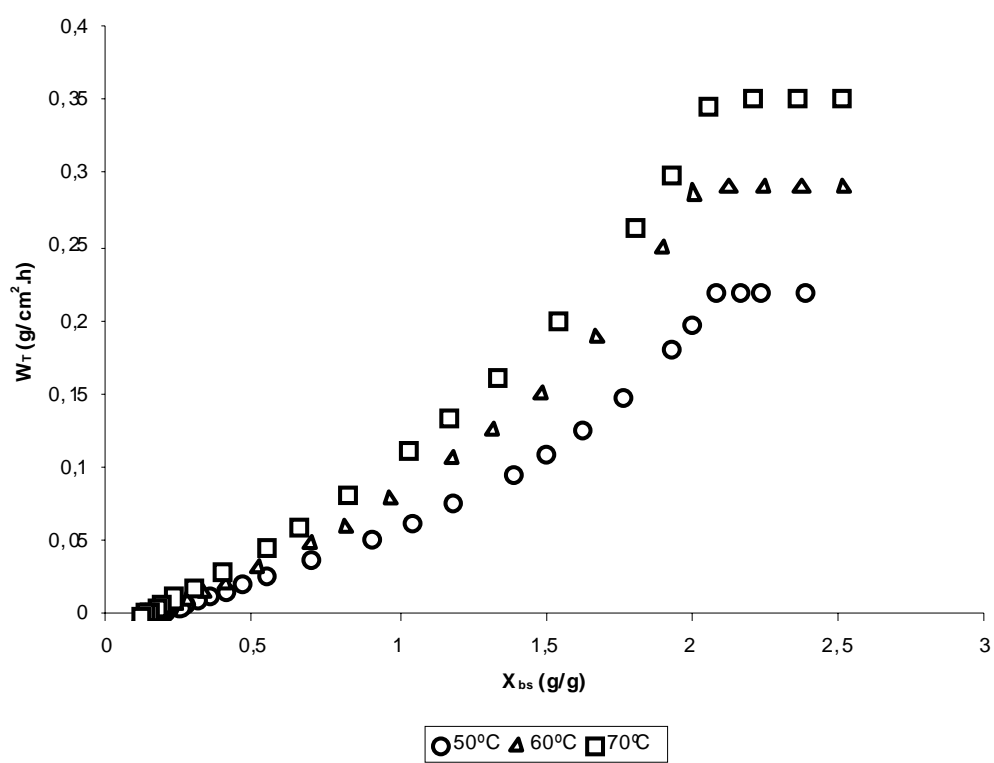




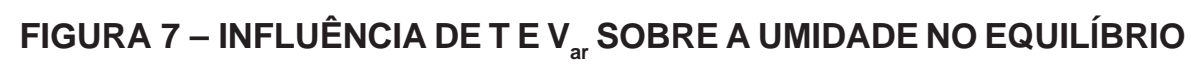

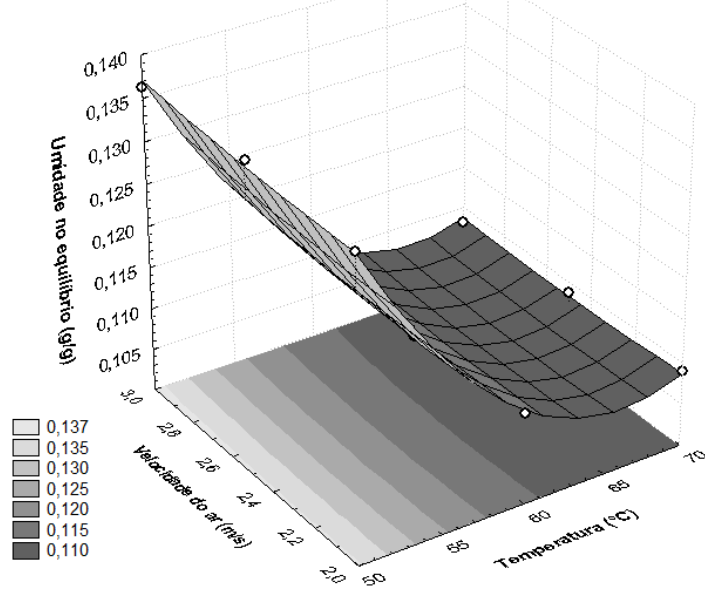

FIGURA 8 - INFLUÊNCIA DE TE V $_{\text {ar }}$ SOBRE O TEMPO DE SECAGEM

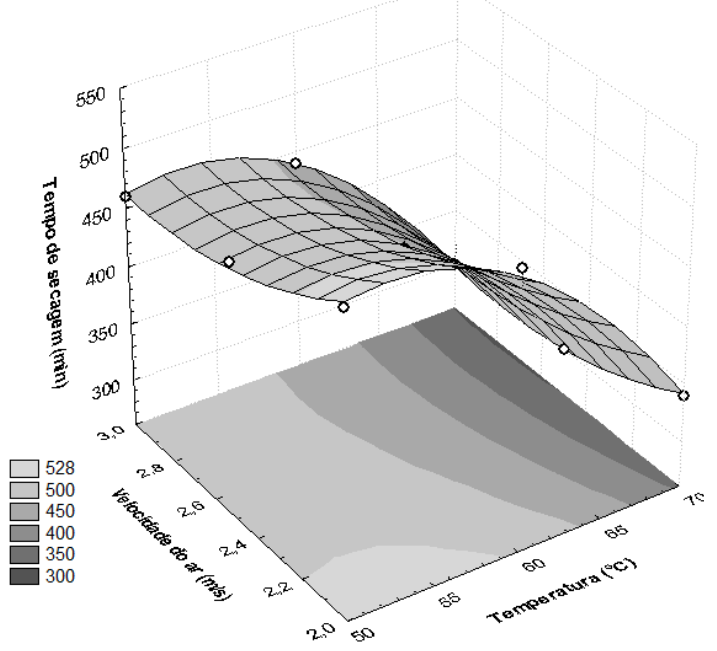

FIGURA 9 - INFLUÊNCIA DE TE $V_{\text {ar }}$ SOBRE A TAXA DE SECAGEM CONSTANTE

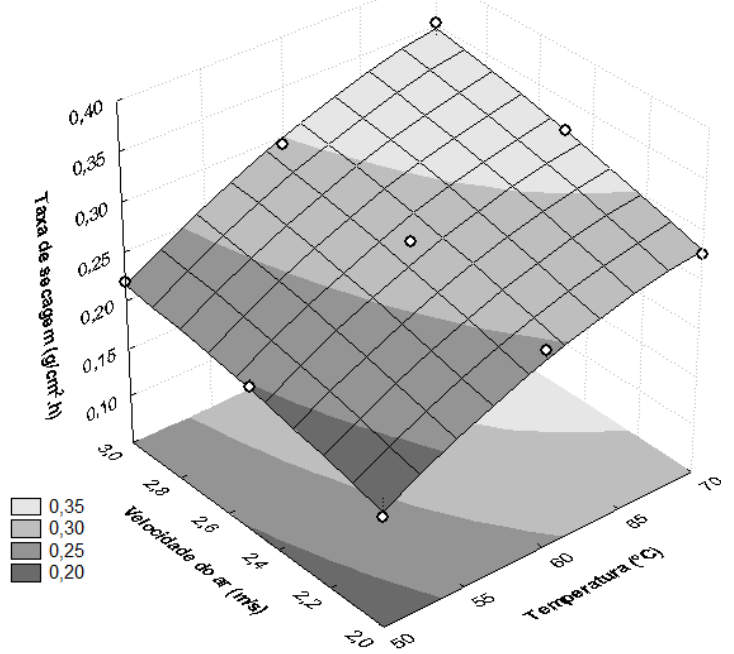




\subsection{AVALIAÇÃO HIGROSCÓPICADO PRODUTO}

\subsubsection{Isotermas de sorção de umidade}

As isotermas de adsorção e dessorção de umidade obtidas a 25 e $40^{\circ} \mathrm{C}$ (Figuras 10 a 13) são do tipo III, de acordo com a classificação da IUPAQ (1985). Segundo SALWIN (1959), esse comportamento é característico de produtos ricos em carboidratos como o resíduo estudado. Observouse ainda o efeito da temperatura e de histerese entre as isotermas de sorção, que se estendeu da região de condensação capilar até a monocamada.

As isotermas de adsorção assumiram comportamento exponencial a partir de 0,5 de $\mathrm{a}_{\mathrm{w}}$, o que indica que o produto requer maiores cuidados quando armazenado em ambiente com umidade relativa superior a 50\%. A adsorção indicou também que o produto alcançará estabilidade microbiológica $\left(a_{w}<0,6\right)$, quando apresentar umidade inferior a $18 \mathrm{~g} / 100 \mathrm{~g}$ b.s., $\mathrm{a} 40^{\circ} \mathrm{C}$.

FIGURA 10 - ISOTERMAS DE ADSORÇÃO DE UMIDADE

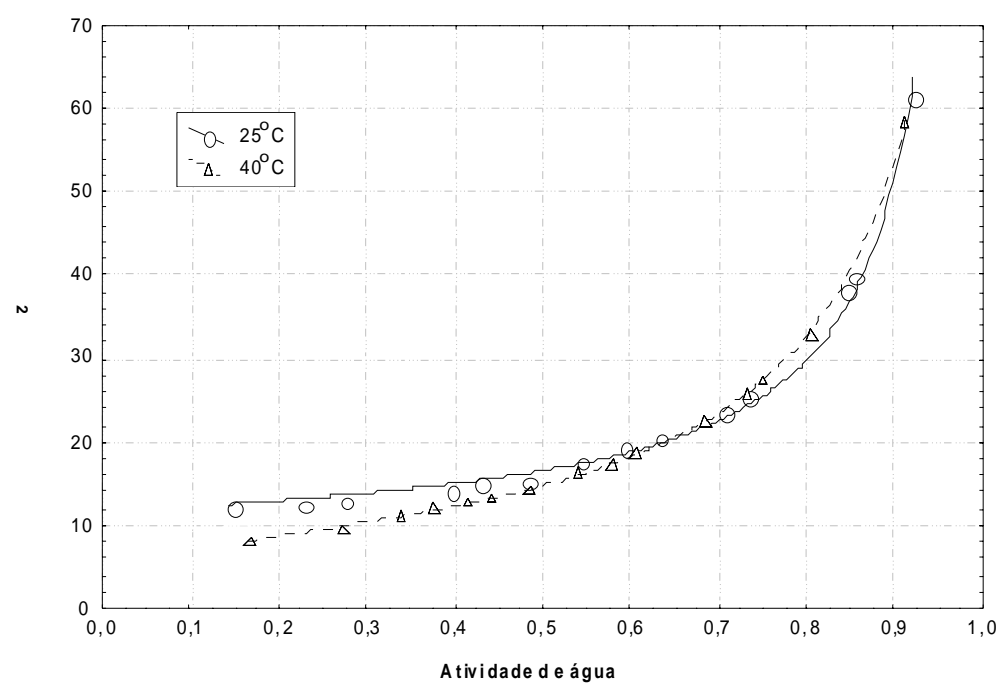

FIGURA 11 - ISOTERMAS DE DESSORÇÃO DE UMIDADE

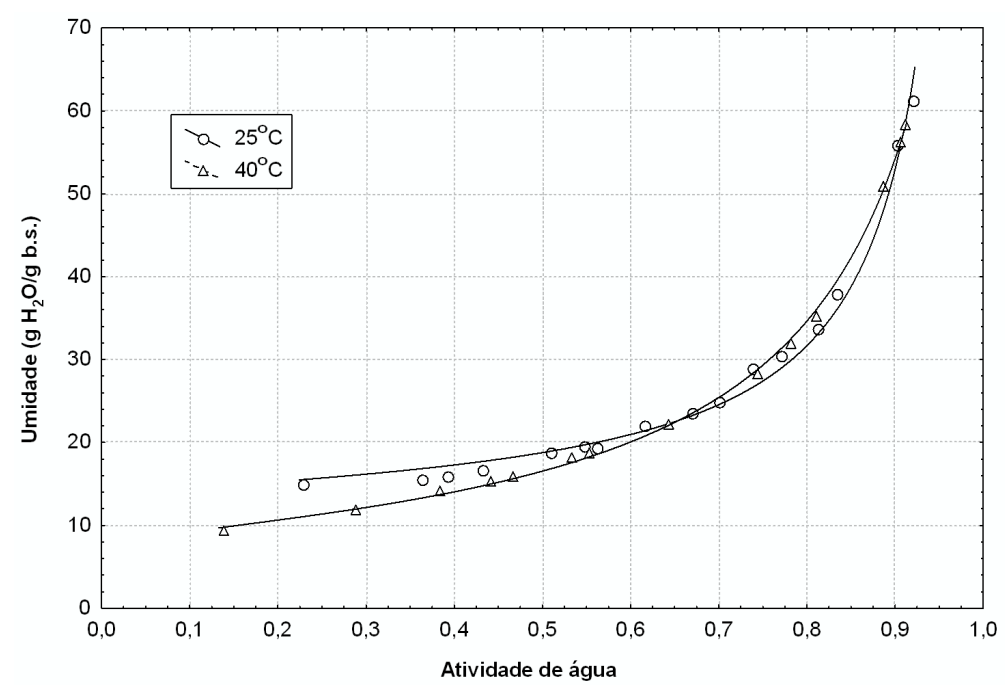




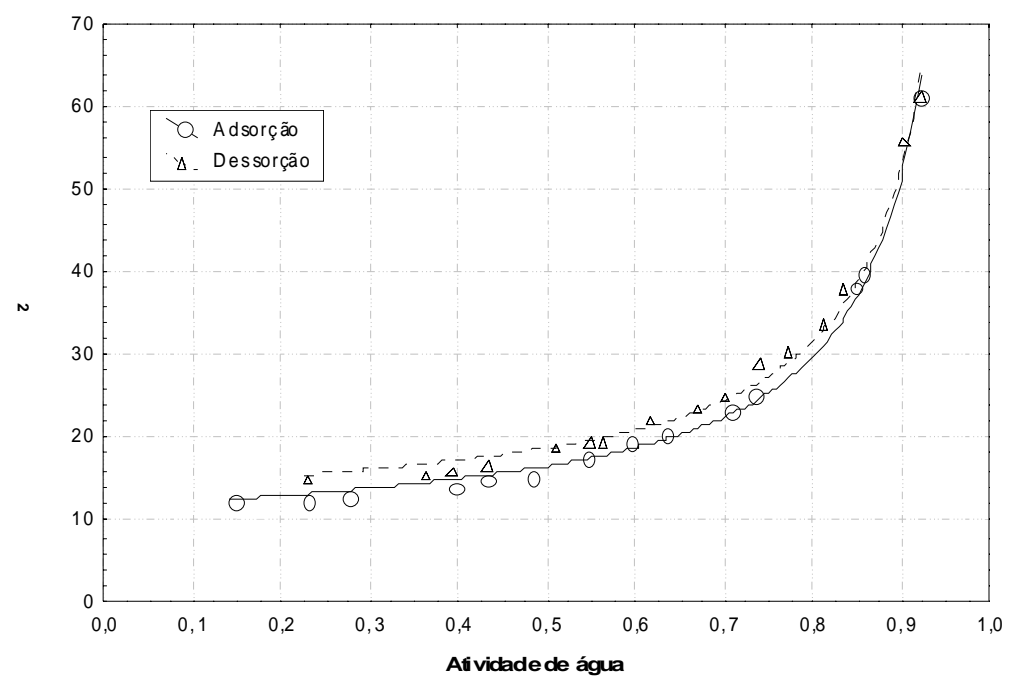

FIGURA 13 - ISOTERMAS DE SORÇÃO A $40^{\circ} \mathrm{C}$

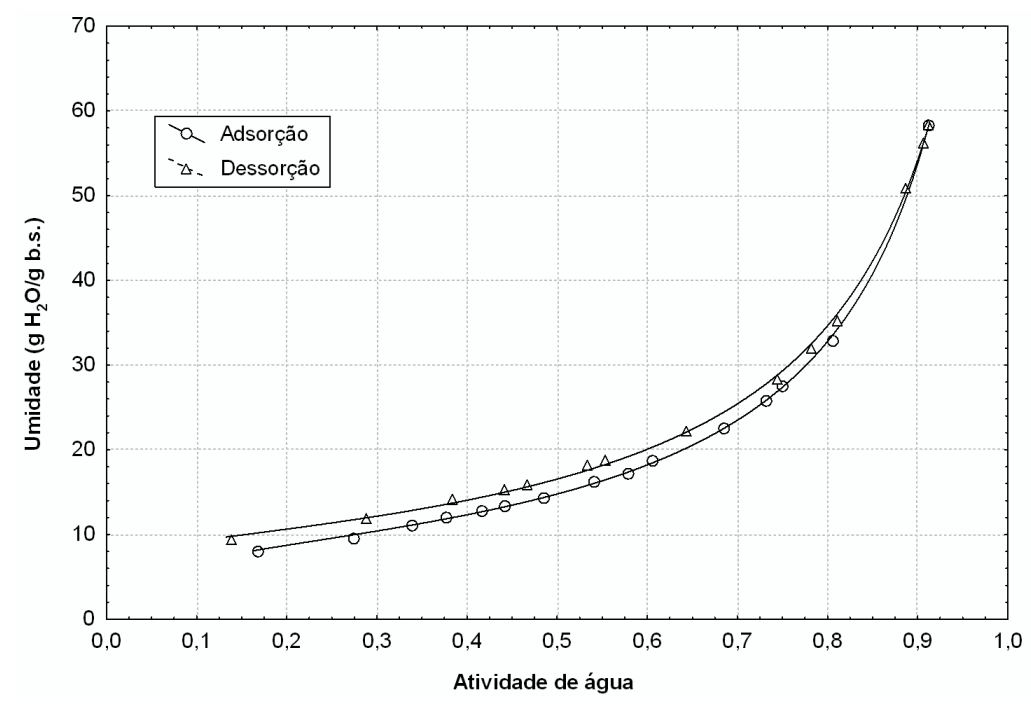

\subsubsection{Determinação da monocamada}

A umidade da monocamada $\left(m_{0}\right)$ para a dessorção, $7,4 \mathrm{~g} \mathrm{H}_{2} \mathrm{O} / 100 \mathrm{~g}$ b.s. a $25^{\circ} \mathrm{C}$ e $8,9 \mathrm{~g} \mathrm{H}_{2} \mathrm{O} /$ $100 \mathrm{~g}$ b.s. a $40^{\circ} \mathrm{C}$, indica que o processo de secagem do produto não deve ser conduzido em níveis de umidade inferiores a 7,4 $\mathrm{g} \mathrm{H}_{2} \mathrm{O} / 100 \mathrm{~g}$ b.s. para evitar gasto desnecessário de energia. Ressalta-se que nesse nível de umidade o produto já apresentará $a_{w}$ inferior a 0,6 , tendo assegurada sua estabilidade microbiológica.

\subsubsection{Determinação do calor de dessorção}

Os calores isostéricos de dessorção, obtidos pela soma do calor latente de vaporização da água $\left(\Delta \mathrm{H}_{v}\right)\left(43,6 \mathrm{~kJ} / \mathrm{mol}\right.$, para a temperatura média de trabalho, $\left.32,5^{\circ} \mathrm{C}\right)$ aos calores líquidos de dessorção, são apresentados na Figura 14. 


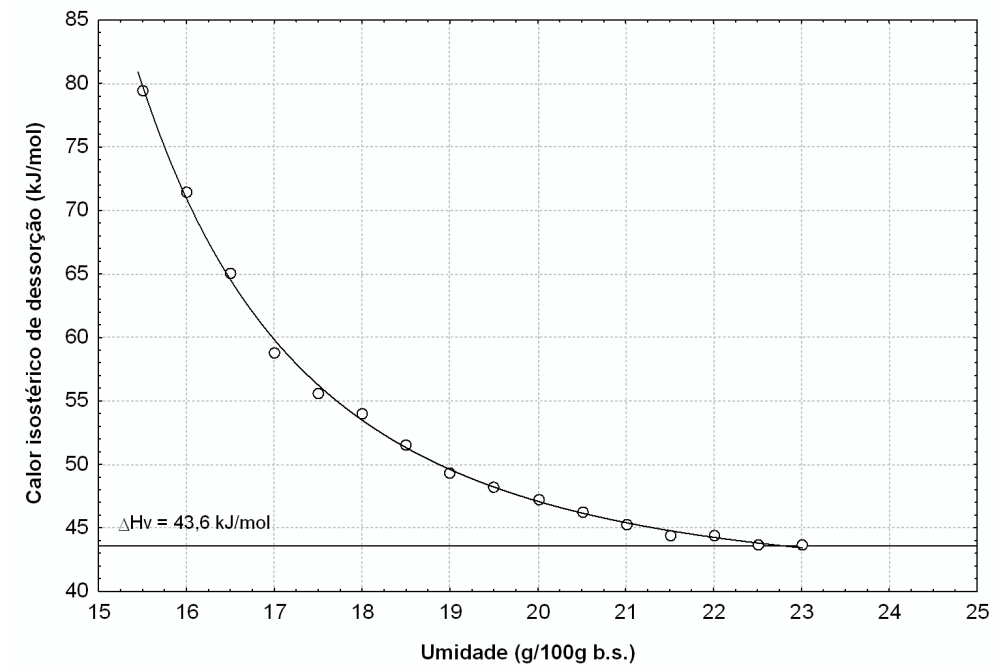

Observou-se que o produto mesmo com 15,5 $\mathrm{g} \mathrm{H}_{2} \mathrm{O} / 100 \mathrm{~g}$ b.s. de umidade, considerada alta para muitos produtos secos, já apresentou calor de dessorção duas vezes superior ao calor latente de vaporização da água. Esse comportamento vem reforçar, mais uma vez, o fato de a maior fração da água presente no resíduo se apresentar combinada aos solutos (açúcares), dificultando sua remoção.

\subsubsection{Predição das isotermas de sorção}

Nas Tabelas 2 e 3 encontram-se, respectivamente, os valores dos coeficientes de determinação $\left(r^{2}\right)$ e desvios médios relativos $(P)$, utilizados para avaliar a modelagem matemática. Com base nos valores de $r^{2}$ e $\mathrm{P}(\mathrm{P}<10 \%)$, constatou-se que as equações de Kuhn, Oswin, Halsey e GAB foram as que apresentaram os melhores ajustes, sendo indicadas para a predição das isotermas de adsorção e dessorção do produto estudado. Chama-se atenção para as equações bi-paramétricas por serem de mais fácil solução matemática, necessitando apenas de regressão linear, especialmente a equação de Halsey por ter apresentado o maior $\mathrm{r}^{2}$ e o menor $\mathrm{P}$.

TABELA 2 - COEFICIENTES DE DETERMINAÇÃO (R²) OBTIDOS NOS AJUSTES

\begin{tabular}{lccccc}
\hline & \multicolumn{2}{c}{ Adsorção } & \multicolumn{2}{c}{ Dessorção } & \\
\cline { 2 - 5 } \cline { 2 - 5 } Equação & $\mathbf{2 5}^{\circ} \mathbf{C}$ & $\mathbf{4 0}^{\circ} \mathbf{C}$ & $\mathbf{2 5}^{\circ} \mathbf{C}$ & $\mathbf{4 0 ^ { \circ } \mathbf { C }}$ & Média \\
\hline Kuhn & 0,9942 & 0,9809 & 0,9907 & 0,9852 & $\mathbf{0 , 9 8 7 8}$ \\
Henderson & 0,9081 & 0,9589 & 0,9272 & 0,9711 & $\mathbf{0 , 9 4 1 3}$ \\
Oswin & 0,9687 & 0,9948 & 0,9768 & 0,9949 & $\mathbf{0 , 9 8 3 8}$ \\
Halsey & 0,9895 & 0,9998 & 0,9905 & 0,9993 & $\mathbf{0 , 9 9 4 8}$ \\
Smith & 0,9329 & 0,9590 & 0,9430 & 0,9772 & $\mathbf{0 , 9 5 3 0}$ \\
GAB & 0,9899 & 0,9997 & 0,9870 & 0,9992 & $\mathbf{0 , 9 9 4 0}$ \\
BET & 0,9631 & 0,9959 & 0,9569 & 0,9919 & $\mathbf{0 , 9 7 7 0}$ \\
& & & & & \\
\hline
\end{tabular}


TABELA 3 - DESVIOS MÉDIOS RELATIVOS (P) OBTIDOS NOS AJUSTES

\begin{tabular}{|c|c|c|c|c|c|}
\hline \multirow[b]{2}{*}{ Equação } & \multicolumn{2}{|c|}{ A dsor ção } & \multicolumn{2}{|c|}{ Desso rção } & \multirow[b]{2}{*}{ Média } \\
\hline & $25^{\circ} \mathrm{C}$ & $40^{\circ} \mathrm{C}$ & $25^{\circ} \mathrm{C}$ & $40^{\circ} \mathrm{C}$ & \\
\hline Kuhn & 4,1 & 8,7 & 3,9 & 8,2 & 6,2 \\
\hline Henderson & 18,3 & 14,2 & 13,6 & 12,6 & 14,7 \\
\hline Oswin & 10,9 & 5,4 & 7,6 & 5,1 & 7,3 \\
\hline Halsey & 6,2 & 1,0 & 4,5 & 1,1 & 3,2 \\
\hline Smith & 13,6 & 11,5 & 10,9 & 9,7 & 11,4 \\
\hline GAB & 6,2 & 1,0 & 5,8 & 1,9 & 3,7 \\
\hline BET & 12,4 & 3,7 & 11,1 & 6,4 & 8,4 \\
\hline
\end{tabular}

\section{CONCLUSÃo}

A fibra residual do maracujá amarelo estudada pode ser alternativamente utilizada na forma de produto seco (farinha), rico em fibra alimentar, obtido pela secagem em camada delgada.

Com base no estudo da secagem do resíduo em camada delgada, recomendam-se como condições de secagem a temperatura de $65^{\circ} \mathrm{C}$ e a velocidade do ar de secagem de $2,5 \mathrm{~m} / \mathrm{s}$. Nessas condições, o produto atingirá umidade de $11 \mathrm{~g} / 100 \mathrm{~g}$ b.s. em, aproximadamente, 6 horas de secagem.

A cinética de secagem e os calores de dessorção mostraram que a maior fração da água presente no resíduo estudado se apresenta na forma ligada, dificultando sua remoção.

O valor da monocamada para a dessorção indicou que o resíduo não deve ser seco à umidade inferior a 7,4 $\mathrm{g} \mathrm{H}_{2} \mathrm{O} / 100 \mathrm{~g}$ b.s. para evitar gasto desnecessário de energia.

As equações de Kuhn, Halsey, Oswin e GAB podem ser utilizadas com excelente precisão na predição da isoterma de sorção do produto, porém recomenda-se a utilização das equações biparamétricas por serem de mais fácil solução matemática e em especial a equação de Halsey.

\section{ABSTRACT}

\section{THIN LAYER DRYING OF RESIDUAL FIBER OF PASSION FRUIT}

It was studied the drying of the residue of the passion fruit (Passiflora edulis) juice processing, in different temperature conditions $\left(50\right.$ to $70^{\circ} \mathrm{C}$ ) and velocity of drying air $(2.0$ to $3.0 \mathrm{~m} / \mathrm{s})$. Aiming to evaluate the hygroscopic behavior of the product, the isotherms of adsorption and desorption at 25 and $40^{\circ} \mathrm{C}$ were obtained. The Kuhn, Henderson, Oswin, Halsey, Smith, GAB e BET equations were fitted to experimental sorption data. The data of adsorption evidenced that the microbiological stability $\left(a_{w}<0,6\right)$ of the product can be insure until $18 \mathrm{~g} \mathrm{H}_{2} \mathrm{O} / 100 \mathrm{~g}$ dry base. The monolayer of desorption indicated that the residue should not be dried to the moisture levels below $7.4 \mathrm{~g} \mathrm{H}_{2} \mathrm{O} / 100 \mathrm{~g}$ dry base, to avoid loss of energy. The heat of desorption evidenced that most water of the product are combined to the solutes. The Kuhn, Oswin, Halsey, GAB and BET equations were found to be the best for predicting the sorption moisture isotherms of the product.

KEY-WORD: Passiflora edulis; PASSION FRUIT - RESIDUE; THIN LAYER DRYING; ISOTHERMS.

\section{REFERÊNCIAS}

1 AKPINAR, E. K. Mathematical modelling of thin layer drying process under open sun of some aromatic plants. Journal of Food Engineering, London, v. 77, n. 4, p.864-870, 2006.

2 AOAC. Association of Official Analytical Chemists. Official methods of analysis of AOAC International. $16^{\text {th }}$ ed. Gaithersburg, Maryland, 1997. 1298 p.

3 ASSUNÇÃO, A. B.; PENA, R. S. Comportamento higroscópico do resíduo seco de camarão-rosa. Ciência e Tecnologia de Alimentos, Campinas, v. 27, n. 4, p. 786-793, 2007.

4 AYROSA A. M. I. B.; MAIZATO M. J. S.; POLAKIEWICZ B., BEPPU M. M.; HIGA O. Z.; PITOMBO R. N. M. Estudo do comportamento higroscópico do pericárdio bovino liofilizado. Revista Matéria, v. 12, n. 2, p. 313-321, 2007.

5 BRASIL. Agência Nacional de Vigilância Sanitária. Portaria nº 27, de 13 de janeiro de 1998. Regulamento técnico referente à informação nutricional complementar. Diário Oficial [da] República Federativa do Brasil, Brasília, 16 de jan. 1998. 
6 BRUNAUER, S.; EMMET, P. H.; TELLER, E. Adsorption of gases in multimolecular layers. Journal American Chemical Society, v. 60, p. 309-319, 1938.

7 BUCKERIDGE, M. S.; TINÉ, M. A. S. Composição polissacarídica: estrutura da parede celular e fibra alimentar. In: LAJOTO, F. M. et al. Fibra dietética em Iberoamérica: tecnologia y salud: obtencion, caracterización, efecto fisiológico y aplicación em alimentos. São Paulo: Varela, 2001. p. 43-60.

8 CHIRIFE, J.; IGLESIAS, H. A. Equations for fitting water sorption isotherms of foods. Part $1-$ a review. Journal of Food Technology, v. 13, p. 159-174, 1978.

9 FERRUA, F. Q.; BARCELOS, M. F. P. Equipamentos e embalagens utilizados em tecnologia de alimentos. Lavras: UFLA/FAEPE, 2003.

10 GEANKOPLIS, C. J. Transport processes and unit operations. $2^{\text {nd }}$ ed. New Jersey: Prentice Hall, 1983.

11 IBGE (Rio de Janeiro, RJ). Produção agrícola municipal 2006. Online. Disponível em: http://<www.ibge.gov.br>. Acesso em: 5 out. 2008.

12 IUPAC. International Union of Pure and Applied Chemistry. Reporting physisorption data for gas/solid systems. Pure and Applied Chemistry, v. 57, n. 4, p. 603-619, 1985.

13 MADAMBA, P. S.; DRISCOLL, R. H.; BUCKLE, K. A. Thin layer drying characteristics of garlic slices. Journal of Food Engineering, Davis, v. 29, n. 1, p. 75-97, 1996.

14 MAROULIS, Z. B.; TSAMI, E.; ARINOS-KOURIS, D.; SARAVACOS, G. D. Application of the GAB model to the sorption isotherms for dried fruits. Journal of Food Engineering, v. 7, p. 63-70, 1988.

15 MARTINAZZO, A. P.; CORRÊA P. C.; RESENDE O.; MELO E. C. Análise e descrição matemática da cinética de secagem de folhas de capim-limão. Revista Brasileira de Engenharia Agrícola e Ambiental, Campina Grande, v.11, n. 3, p. 301-306, 2007.

16 McCABE, W. L.; SMITH, J. C.; HARRIOTT, P. Unit operations of chemical engineering. $5^{\text {th }}$ ed. New york: McGraw-Hill, 1993.

17 OKADA, M. Fundamentos sobre secagem de sólidos. In: TRAVAGLINI, D. A. (Coord.). Desidratação de frutas e hortaliças. Campinas: ITAL, 1987. p. 1-38.

18 OLIVEIRA, L. F.; NASCIMENTO, M. R. F.; BORGES, S. V.; RIBEIRO, P. C. N.; RUBACK, V. R. Aproveitamento alternativo da casca do maracujá-amarelo (passiflora edulis. F. Flavicarpa) para produção de doce em calda. Ciência e Tecnologia de Alimentos, Campinas, v. 22, n. 3, p. 259-262, set./dez. 2002.

19 PARK, K. J.; NOGUEIRA, R. I. Modelos para ajuste de isotermas de sorção de alimentos. Engenharia Rural, Piracicaba, v. 3 , n. 1 , p. $80-6,1992$.

20 PENG, G.; CHEN, X.; WU, W.; JIANG, X. Modeling of water sorption isotherm for corn starch. Journal of Food Engineering, v. 80, n. 2, p. 562-567, 2007.

21 RUGGIERO, C. (Coord.). Maracujá para exportação: aspectos técnicos. Brasília: EMBRAPA-SPI, 1996. p.11-29.

22 SABBAG, O. J.; TARSITANO, M. A.; CORRÊA, L. S. Análise da produção e comercialização do maracujá amarelo (Passiflora edullis f. flavicarpa) no mercado brasileiro. In: CONGRESSO BRASILEIRO DE FRUTICULTURA, 17., 2002, Belém, PA. Anais... Belém: SBF/EMBRAPA, 2002.

23 SALWIN, H. Defining minimum moisture contents for dehydrated foods. Food Technology, v. 13, p. 594-585, 1959.

24 SILVA, A. E.; SILVA, L. H. M.; PENA, R. S. Comportamento higroscópico do açaí e cupuaçu em pó. Ciência e Tecnologia de Alimentos, Campinas, v. 28, n. 4, p. 895-901, 2008.

25 SILVA, H. L. B. Elaboração de bebidas lácteas adicionadas de xarope do resíduo de maracujá obtido da centrífuga. 2002. Trabalho de Conclusão de Curso (Especialização em Tecnologia de Alimentos) - Universidade Federal do Pará, Belém, 2002.

26 SOUZA, A. C. G; SANDI, D. Industrialização. In: BRUCKNER, C.H.; PICANÇO,M.C. (ed.) Maracujá: tecnologia de produção, pós colheita, agroindústria, mercado. Porto Alegre: Cinco Continentes, 2001. p. 305-343.

27 STATISTICA for Windows 5.5. Computer program manual. Tulsa: StatSoft Inc., 2000.

28 WAUGHON, T. G.; PENA, R. S. Modelagem da secagem em camada delgada da fibra residual do abacaxi. Boletim do Centro de Pesquisa e Processamento de Alimentos, Curitiba, v. 26, n. 2, p. 297-306, 2008.

\section{AGRADECIMENTO}

À Financiadora de Estudos e Projetos (FINEP) pelo apoio financeiro indispensável à execução deste trabalho. 